\title{
Gut microbiota and obesity: implications for fecal microbiota transplantation therapy
}

\author{
Yongbo Kang, ${ }^{1,2, *}$ Yue Cai ${ }^{1,3, *}$ \\ ${ }^{1}$ Medical Faculty, Kunming University of Science and Technology, Kunming, Yunnan, China; ${ }^{2}$ Genetics and Pharmacogenomics \\ Laboratory, Kunming University of Science and Technology, Kunming, Yunnan, China; ${ }^{3}$ Pathogen biology Laboratory, \\ Kunming University of Science and Technology, Kunming, Yunnan, China
}

*Equal contributors

\begin{abstract}
Obesity is a major public health issue as it is causally associated with several chronic disorders, including type-2 diabetes, cerebrovascular disease (CVD), and cancer. In the United States and other countries worldwide, the obesity epidemic has drastically impacted the status of health of millions as well as healthcare costs. Aside from poor diet, hygiene, and genetics, there are many other factors thought to play an important role in the emergence of obesity. Nowadays, accumulating evidence is elucidating the relation of dysbiosis of intestinal bacteria with obesity and metabolic disorders. Certain gut microbial strains have been shown to inhibit or attenuate immune responses related to chronic inflammation in experimental models, suggesting that specific species among gut microbiota may play either a protective or a pathogenic role in the progression of obesity. Fecal microbiota transplantation (FMT) can therefore represent a therapeutic approach for obesity treatment. FMT is a relatively straightforward therapy that manipulates the human gastrointestinal (GI) microbiota by transferring healthy donor microbiota into an existing but disturbed microbial ecosystem. However, the relevant scientific work is still in its early stages. In this review, we summarize the cutting-edge research being done into FMT treatment of obesity, current issues in FMT treatment, and the future of FMT and microbial therapeutics.
\end{abstract}

Key words: Fecal microbiota transplantation, Gut microbiota, Metabolic syndrome, Obesity

\section{INTRODUCTION}

Obesity, today a huge public health issue, ${ }^{1}$ is mainly a disequilibrium in energy balance, with energy intake

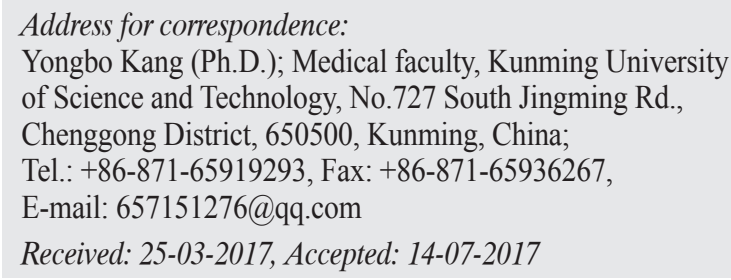

exceeding energy expenditure, a condition characterized by a state of chronic inflammation. Obesity is a problem not only in terms of appearance but also as a trigger for development of metabolic diseases such as type 2 diabetes, cardiovascular diseases, osteoarthritis, and certain cancers. ${ }^{2}$ At present, there are more than 500 million adults worldwide who are overweight [body mass index (BMI) of $25.0-29.9 \mathrm{~kg} / \mathrm{m}^{2}$ ] and 250 million that are obese $\left(\mathrm{BMI} \geq 30 \mathrm{~kg} / \mathrm{m}^{2}\right)$. More than $50 \%$ of adults in the United States, Canada, and 
several other countries have a BMI of at least 25.0 $\mathrm{kg} / \mathrm{m} .^{2,3}$ It is, in other words, a growing epidemic that is threatening numerous countries round the globe. At the same time, obesity-related disorders are also on the rise worldwide.

However, changes in energy balance alone cannot explain the increased incidence of obesity. ${ }^{4}$ Recent studies in animal models and human subjects have revealed that dysbiosis of the gut microbiota is also associated with the development of obesity. Change in the abundance of certain gut microbial strains has been shown to inhibit or attenuate immune responses giving rise to chronic inflammation in experimental models: these strains may be biomarkers in obesity prevention and treatment. On the other hand, though still a relatively nascent field of research, evidence to date suggests that the gut microbiome may represent fertile targets for prevention or management of obesity, with the result that the development of therapeutics based on gut microbiota modulation has gained considerable momentum. Fecal microbiota transplantation (FMT) represents a feasible and easy therapy for treating, and possibly even curing, obesity by influencing the abundance and functions of several of the intestinal microbiota and regulating several pathogenic alterations. In this review, we summarize the recent literature as an aid to better understanding how the alteration of gut microbiota composition contributes to obesity while evaluating the therapeutic effect of FMT in the treatment of obesity based on previous publications.

\section{GUT MICROBIOTA: DEFINITION}

The intestinal epithelium, mucosal immune system, and microflora constitute a morphofunctional system in dynamic equilibrium that is responsible for the maintenance of gut integrity and systemic barrier function. ${ }^{5}$ The human intestine harbors a large diversity of microbial communities, which are collectively termed the gut microbiota. An ecologic unit harboring $10^{14}$ bacteria,${ }^{6}$ it consists of 6-10 major phyla and 30005000 species weighing $1-2 \mathrm{~kg} .{ }^{6}$ The dominant phyla in the gut are Firmicutes (60-65\%), Bacteroidetes (2025\%), Proteobacteria (5-10\%), and Actinobacteria $(3 \%)$, which together constitute over $97 \%$ of the gut microbe population. ${ }^{7}$ Its very diversity sheds light on the role of these different bacterial species in the maintenance of health and the development of disease. Although the number of microbial luminal cells is close to that of eukaryotic cells of living beings, ${ }^{8}$ the collective genome of the microbiota (known as the microbiome) contains 100 -fold more genes than the entire human genome. ${ }^{9}$ The microbial metagenome can provide a huge genetic diversity capable of exerting a large number of functions. Complex microbial communities play a crucial role in the health of the human organism and these highly evolved lineages have evolved with humans over the eons. ${ }^{10}$ The gastrointestinal tract is essentially sterile at birth and is subsequently colonized by microbes acquired from the mother and the surrounding environment. ${ }^{11}$ The gut microbiota also undergoes substantial alterations at the extremes of life, that is, in infants and in older people. ${ }^{12}$ However, adult gut microbiota has been shown to be comparatively steady over time ${ }^{13}$ and is relatively alike between individuals even when there are drastic changes in diet and in levels of physical activity, during pregnancy, and with the use of broadspectrum antibiotics. ${ }^{14,15}$

The gut microbiota digest nutrients, synthesize vitamins, butyric acid and biotin, and degrade bile acid and short-chain fatty acids (SCFAs), including acetate, propionate, and butyrate. ${ }^{16}$ Specifically, in addition to their important role as fuel for intestinal epithelial cells, SCFAs are thought to have immunomodulatory effects in that they reduce intestinal permeability. ${ }^{17}$ SCFAs also regulate different processes of the gastrointestinal (GI) tract (e.g. water absorption and electrolytes)..$^{18}$ Gut microbiota can impact intestinal immune function through SCFAs, and these fatty acids act on leukocytes and endothelial cells via two mechanisms: (1) by activating $G$ protein coupled receptors (GPCRs) (GPR41 and GPR43) and (2) by inhibiting histone deacetylase (HDAC) ${ }^{19}$ SCFAs not only modulate several leukocyte functions, including production of chemokines such as CINC-2 and MCP1, eicosanoids and cytokines (e.g. IL-10, IL-6, IL-2, and TNF- $\alpha),{ }^{20}$ but also affect the ability of leukocytes to migrate to the foci of inflammation and to destroy microbial pathogens. ${ }^{18,21}$ Furthermore, SCFAs can also lead to apoptosis of neutrophils, ${ }^{22}$ macrophages,${ }^{23}$ and lymphocytes. ${ }^{24,25}$ The effect may come about as the result of the inflammatory process and the immune 
response to gut microbiota, which produce SCFAs, these capable of exerting an anti-inflammatory action. Meanwhile, a large amount of recent research has convincingly demonstrated that the stability and diversification of gut microbia is essential for the hosting of metabolic and immune pathways. ${ }^{26}$ The gut is a crucial immune organ besides its function in metabolism, while it contains the largest lymphoid tissue mass, ${ }^{27}$ which might not be surprising considering its intestinal bacterial load. Thus, in addition to contributing to host metabolism, the gut microbiota provides critical signals for the development of host immunity. Hence, gut microbes are crucial for the maintenance of human health and the treatment of disease. Conversely, disruptions of this intricate balance between host and commensal microbes have dramatic effects on human health and disease.

\section{GUT MICROBIOTA AND OBESITY}

Experimental models have revealed several mechanisms connecting the gut microbiota to obesity and metabolic disorders. The proposed mechanisms by which the microbiome may contribute to the development of obesity include: 1) increasing dietary energy harvest, 2) promoting fat deposition, 3) possibly modifying locomotor activity, 4) having central effects on satiety, 5) and triggering systemic inflammation (Figure 1). ${ }^{28}$ The vast majority of these studies suggest that subjects with obesity exhibit alterations in the relative abundance of "beneficial" and potentially "harmful" bacteria compared to healthy subjects (Table 1). A link between the gut microbiota and obesity was initially demonstrated based on studies in germ-free (GF) mice. ${ }^{29}$ By comparing them to conventionally raised mice, it was observed that these GF mice had significantly less total body fat even if they consumed markedly more food. Furthermore, fecal microbiota transplantation from normal mice into the GF mice led to a significant increase in total body fat and insulin resistance without any increase in food consumption or changes in energy expenditure. In keeping with the latter observation, Bäckhed et $\mathrm{al}^{30}$ found that compared to their normal counterparts, GF mice are protected from development of obesity after consuming a Western-style, high-fat, sugar-rich diet. Their persistently lean phenotype is associated with increased skeletal muscle and liver levels of phosphorylated AMP-activated protein kinase (AMPK) and its downstream targets involved in fatty acid oxidation (acetylCoA carboxylase; carnitine palmitoyltransferase). In addition, fasting-induced adipose factor (Fiaf), which belongs to a member of the angiopoietin family of proteins, is a circulating lipoprotein lipase inhibitor and is normally selectively suppressed in the intestinal epithelium by gut microbiota. GF Fiaf-/-mice were not protected against diet-induced obesity. Although GF Fiaf-/-mice had similar gastrocnemius muscle and liver levels of phosphorylated AMPK to their wild-type littermates, they exhibited decreased expression of the genes encoding the enzymes regulating fatty acid oxidation and of the genes encoding the peroxisome proliferator-activated receptor gamma $(\gamma)$ coactivator 1-alpha (PGC-1 $\alpha)$. Moreover, another study demonstrated that GF mice colonized with fecal microbiota from obese $o b / o b$ mice had a significant increase in dietary caloric extraction and fat gain compared to microbiota from lean $(\mathrm{ob} /+$ or $+/+)$ mice, ${ }^{31}$ this suggesting that intestinal bacteria influence the phenotypic characteristics of the host. Furthermore, genetically obese $o b / o b$ mice are hyperphagic due to a mutation in the gene encoding the satiety-promoting hormone leptin..$^{32}$ These mice have a significant increase of Firmicutes and a marked decrease of Bacteroidetes compared to their lean wild-type littermates, even when the mice are fed the same low-fat, polysaccharide-rich diet. ${ }^{32}$ Changes of intestinal microbiota may affect the development of obesity and peripheral organs involved in the control of satiety in the brain. Taken together, these data demonstrated that gut commensals can influence satiety and the energy harvest as well as fat deposition.

A growing body of evidence has revealed that low-grade chronic systemic inflammation is related to obesity and insulin resistance..$^{33}$ Bacterial lipopolysaccharide (LPS) derived from intestinal bacteria can induce systemic inflammation by binding to CD14 receptors. ${ }^{34}$ Cani et al ${ }^{35}$ demonstrated that mice fed a high-fat diet had a significant increase of plasma LPS levels compared with mice fed a normal diet. They also showed that chronic metabolic endotoxemia may lead to adiposity and insulin resistance. A previous study reported that antibiotics can reduce LPS, tumor necrosis factor (TNF) production, and 


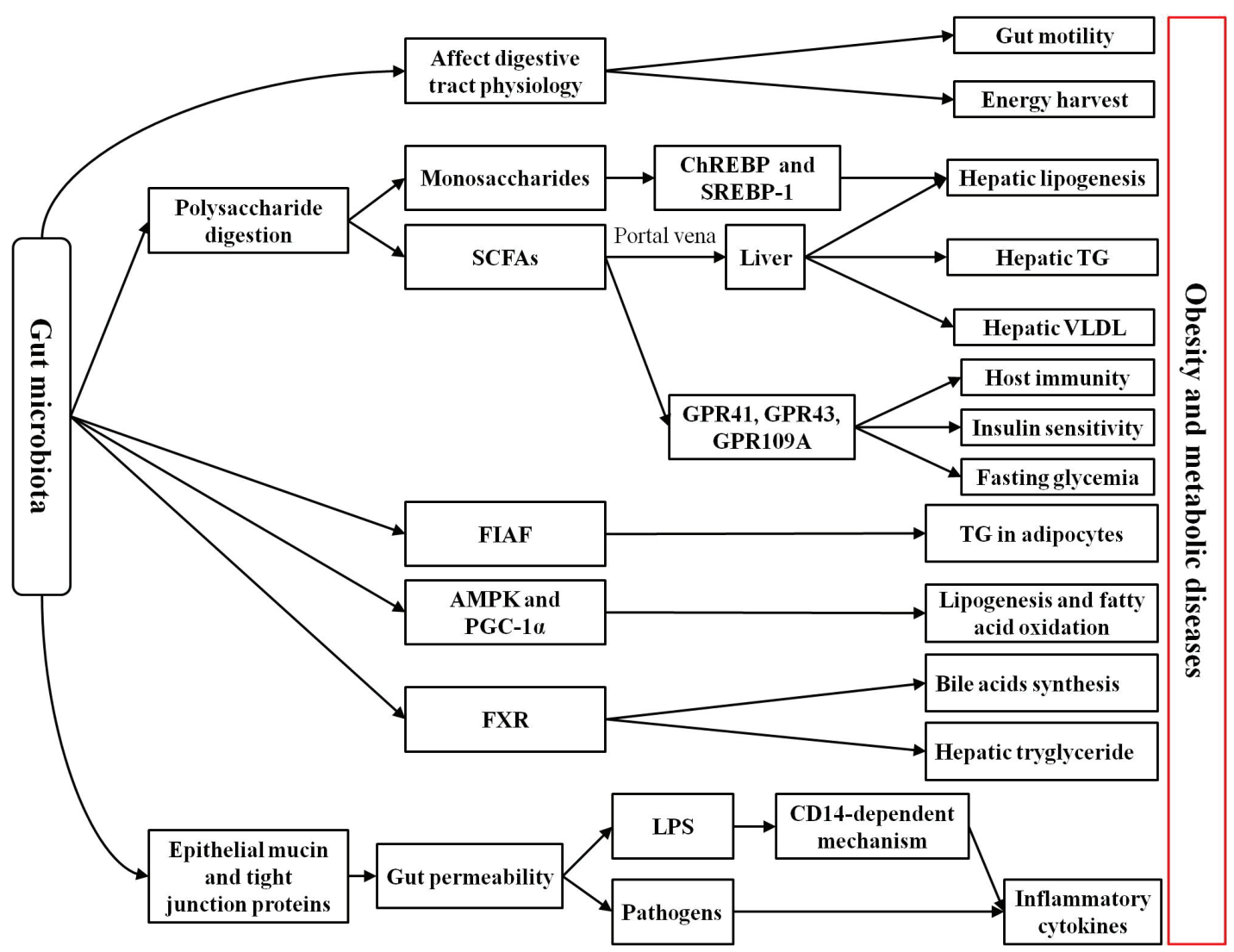

Figure 1. Possible mechanisms associated with the relationship between intestinal microbiota and obesity. Intestinal microbiota is thought to participate in molecular crosstalk with the host that modulates host physiology, metabolism, gut barriers, and inflammatory status. In particular, intestinal microbiota is involved in the physiology and motility of the digestive tract and in the digestion of polysaccharides, which could directly affect host energy availability. The monosaccharides produced by the intestinal microbiota from polysaccharides is likely to increase hepatic lipogenesis via the carbohydrate response element binding protein (ChREBP) and sterol response element-binding protein type 1 (SREBP-1). The short-chain fatty acids (SCFAs) produced by the intestinal microbiota from polysaccharides interact with G protein-coupled receptors (GPCRs; GPR41, GPR43, and GPR109A), which might be implicated in activating host immunity, regulating fasting glycemia, and affecting insulin sensitivity. Furthermore, SCFAs transported to the liver possibly increase production of hepatic lipogenesis, hepatic triglycerides (TG), and very low density lipoproteins (VLDL). The intestinal microbiota inhibits fasting-induced adipose factor (Fiaf) and adenosine monophosphate-activated protein kinase (AMPK) peroxisome proliferator-activated receptor $\gamma$ coactivator $1 \alpha(\mathrm{PGC}-1 \alpha)$, which may lead to increasing fat deposition. The intestinal microbiota also contributes to fat deposition through the regulation of the farnesoid X receptor (FXR), the bile acid receptor responsible for the regulation of bile acid synthesis and hepatic triglyceride accumulation. The gut microbiota also contributes to increasing intestinal permeability via decreasing the epithelial mucin and tight junction proteins, which could result in translocation of pathogens and lipopolysaccharides (LPS) across the intestinal wall. In addition, pathogens and LPS (via a CD14-dependent mechanism) may induce production of inflammatory cytokines.

hepatic steatosis. ${ }^{36}$ Similar metabolic features were found in CD14 knockout mice. In conclusion, the above data demonstrate that commensal bacteria are involved in systemic inflammation through the LPS/ CD14 system. ${ }^{35}$

Obesity has further been shown to be related to altered gut microbial abundance and composition in human subjects ${ }^{37,38}$ and mice. ${ }^{32}$ Most $^{31,38}$ but not all ${ }^{39}$ studies reported on the diversity of the microbiota and particularly underlined the decrease in fractional proportion of Bacteroidetes species relative to Firmicutes in obese compared with lean individuals..$^{31,38-40}$ Meanwhile, weight loss was shown to increase the relative proportion of Bacteroidetes species and microbial diversity. ${ }^{37,41}$ Similarly, diversity of the genes was decreased in obese compared with lean individu- 
Table 1 Changes in microbiota composition associated with obesity.

\begin{tabular}{lllc}
\hline Models & Disease & Implicated microbiota & Reference \\
\hline Rat & Obesity & Altered gut microbiota & 29,30 \\
Rat & Obesity & Firmicutes $\uparrow$, Bacteroidetes $\downarrow$ & 31,32 \\
Human & Obesity & Firmicutes $\uparrow$, Bacteroidetes $\downarrow$ & $37,38,39,40,41$ \\
Human & Obesity & Proteobacteria $\uparrow$, Bacteroidetes $\uparrow$, Campylobacter $\uparrow$, Shigella $\uparrow$, Lactobacillus $\uparrow$, & 43,44 \\
& & Clostridium coccoides $\uparrow$, Bifidobacterium $\uparrow$, Akkermansia muciniphila $\downarrow$, & 46 \\
Human & Clostridium leptum $\downarrow$, Bacteroides fragilis $\downarrow$, Bifidobacterium catenulatum $\downarrow$ & 48 \\
Human & Obesity & H2-producing Prevotellaceae $\uparrow$, H2-utilizing methanogenic Archaea $\uparrow$ & 48 \\
\hline
\end{tabular}

als $^{38}$ and was increased by dietary weight loss. ${ }^{42,43}$ Recently, Le Chatelier et $\mathrm{al}^{44}$ also found that obese subjects had an increase of the phyla Proteobacteria and Bacteroidetes, a decrease of Akkermansia muciniphila (anti-inflammatory bacteria), and an increase of pathogens (e.g. Campylobacter and Shigella). ${ }^{44}$ These alterations in intestinal bacteria can diminish intestinal barrier integrity and increase oxidative stress and mucus degradation through reducing the production of butyrate. ${ }^{44}$ Another study demonstrated that $A$. muciniphila can reduce the insulin resistance index and control fat storage, adipose tissue metabolism, intestinal levels of acylglycerols, and glucose homeostasis. ${ }^{45}$ The increased abundance of Clostridium leptum, Bacteroides fragilis, and Bifidobacterium catenulatum and decreased abundance of Lactobacillus, Clostridium coccoides, and Bifidobacterium after dietary interventions were strongly correlated with significant weight loss, regardless of total food intake. ${ }^{43,44}$ In accord with the above observation, Zhang et $\mathrm{al}^{46}$ reported that the levels of $\mathrm{H}_{2}$-producing bacterial groups, comprised mainly of members of the Prevotellaceae family and certain groups within Firmicutes and $\mathrm{H}_{2}$-oxidizing methanogenic Archaea, were significantly higher in the gastrointestinal tracts of obese subjects compared to healthy subjects. As $\mathrm{H}_{2}$-producing bacteria coexist with $\mathrm{H}_{2}$-oxidizing methanogenic Archaea in the gastrointestinal tracts, the authors put forward the hypothesis that the interspecies $\mathrm{H}_{2}$ transfer between bacterial and archaeal species had an impact on energy handling. ${ }^{46}$ Methanogens absorb $\mathrm{H}_{2}$ rapidly, which can accelerate the fermentation of carbohydrates by $\mathrm{H}_{2}$-producing bacterial groups. ${ }^{46}$ Meanwhile, the accelerated fermentation can increase the hydrolysis efficiency of usually indigestible organic matter. ${ }^{47}$
Recently, similar data were reported by Fei and Zhao. ${ }^{48}$ It should be noted that lipopolysaccharide endotoxin is the only known bacterial product, which, when subcutaneously infused into mice in its purified form, can induce obesity and insulin resistance via an inflammation-mediated pathway. ${ }^{49}$ Enterobacter, a genus of opportunistic endotoxin-producing pathogens,${ }^{50}$ made up $35 \%$ of the gut bacteria in a morbidly obese volunteer (weight $174.8 \mathrm{~kg}$, BMI $58.8 \mathrm{~kg} / \mathrm{m}^{2}$ ) suffering from diabetes, hypertension, and other serious metabolic disorders. After 23 weeks on a diet of whole grains, traditional Chinese medicinal foods, and prebiotics (WTP diet), the Enterobacter decreased in relative abundance from $35 \%$ of the volunteer's gut bacteria to nondetectable. ${ }^{48}$ In addition, during this time the volunteer lost 51.4 of $174.8 \mathrm{~kg}$ initial weight and his hyperinsulinemia, hyperglycemia, and hypertension resolved. ${ }^{48}$ Thus, alterations of intestinal bacteria play a key role in ensuring the efficacy of obesity treatments, with specific changes of the commensal microbes representing a potential therapy to promote and sustain weight loss and/or to prevent adiposity in humans. However, further studies are necessary to test the efficacy and safety of using interventions involving prebiotics, probiotics, and FMT before they are implemented in clinical routine.

\section{FECAL MICROBIOTA TRANSPLANTATION THERAPY IN OBESITY}

Fecal microbiota transplantation is the administration of a solution of fecal matter from a donor into the intestinal tract of a recipient in order to directly alter the recipient's microbial composition and confer a health benefit. ${ }^{51,52}$ The process usually involves 
first selecting a donor without a family history of autoimmune, metabolic, and malignant diseases and screening for any potential pathogens. The feces are then prepared by mixing with water or normal saline, followed by a filtration step to remove any particulate matter. The mixture can be administered through a nasogastric tube, nasojejunal tube, esophagogastroduodenoscopy, colonoscopy, or retention enema. ${ }^{53}$ Although FMT has been utilized for over 50 years, it has recently gained momentum given its high efficacy in eradicating Clostridium difficile infection. ${ }^{54}$ Most clinical experience with FMT has been derived from treating recurrent or refractory Clostridium difficile infection (CDI) ${ }^{52}$ Recently there have been several trials evaluating the prospect of altering the gut microbiome as a potential for therapy in obesity and the metabolic syndrome (Table 2). In this context, possible mechanisms of action of FMT in such situations include manipulation of the composition of gut microbiota, fortification of the intestinal barrier and suppression of pathogens, and immunomodulation (Figure 2). ${ }^{55} \mathrm{~A}$ large body of research has demonstrated that alteration of the relative level of the two dominant bacterial divisions (Bacteroidetes and Firmicutes) was related to obesity, ${ }^{29-32}$ namely, the abundance of Bacteroidetes was lower, while the abundance of Firmicutes was higher in obese individuals. Meanwhile, the obese microbiome was shown to be more efficient at absorbing energy from food by digestion compared to the lean microbiome. ${ }^{31}$ Turnbaugh et $\mathrm{al}^{31}$ completed a more in-depth study than the previous research, in which the fecal micro- biota of obese $\mathrm{C} 57 \mathrm{BL} / 6 \mathrm{~J}$ donors was transplanted into germ-free $\mathrm{C} 57 \mathrm{BL} / 6 \mathrm{~J}$ recipients. Germ-free mice colonized with an 'obese microbiota' exhibited a significant increase in total body fat compared with germ-free mice colonized with a 'lean microbiota'. Furthermore, the obese recipient microbiota had a significantly higher level of Firmicutes than the lean recipient microbiota. These results showed intestinal bacteria to be an additional contributing factor to the pathophysiology of obesity. In 2012, Vrieze et al ${ }^{56}$ evaluated the effects of FMT on insulin sensitivity in subjects with metabolic syndrome. Patients with metabolic syndrome were randomly assigned to groups that were given small intestinal infusions of allogenic or autologous microbiota. Patients who received an infusion of microbiota from lean donors were observed to have a significant increase in insulin sensitivity and butyrate-producing intestinal microbiota (e.g. Eubacterium hallii or Roseburia intestinalis). Butyrate as a short-chain fatty acid is known to be associated with obesity and pain sensation and plays an important role in colonic function. ${ }^{57-60}$

Thus, gut microbiota could be developed as therapeutic agents to increase insulin sensitivity in humans. Ridaura et a ${ }^{61}$ transplanted fecal microbiota from adult female twin pairs discordant for obesity (an obese or lean co-twin) into separate groups of GF mice fed mouse chow low in fat and rich in plant polysaccharides. Mice that received an obese twin's fecal microbiota exhibited significantly greater increases in total body, fat mass and adiposity, and obesity-associated

Table 2. Changes in microbiota composition associated with obesity and FMT therapeutic strategies

\begin{tabular}{|c|c|c|c|c|c|}
\hline Models & Disease & Implicated microbiota & $\begin{array}{l}\text { New therapeutic } \\
\text { strategies }\end{array}$ & Implicated microbiota & Reference \\
\hline Mice & Obesity & Bacteroidetes $\downarrow$, Firmicutes $\uparrow$ & FMT & Bacteroidetes $\uparrow$, Firmicutes $\downarrow$ & 31 \\
\hline Human & $\begin{array}{l}\text { Metabolic } \\
\text { syndrome }\end{array}$ & NO & FMT & $\begin{array}{l}\text { Butyrate-producing microbiota (e.g. Roseburia } \\
\text { intestinalis or Eubacterium hallii) }\end{array}$ & 56 \\
\hline Mice & Obesity & NO & FMT & NO & 61 \\
\hline Mice & Obesity & $\begin{array}{c}\text { Gammaproteobacteria } \\
\text { (Escherichia) } \downarrow \text {, Verrucomicrobia } \\
\text { (Akkermansia) } \downarrow \text { and } \\
\text { Bacteroidales } \downarrow\end{array}$ & FMT & $\begin{array}{c}\text { Verrucomicrobiales } \uparrow, \text { Akkermansia } \uparrow, \\
\text { Alistipes (Bacteroidetes phylum) } \uparrow\end{array}$ & 65 \\
\hline Mice & Obesity & NO & FMT & NO & 66 \\
\hline
\end{tabular}

NO refers to no test or no research. 


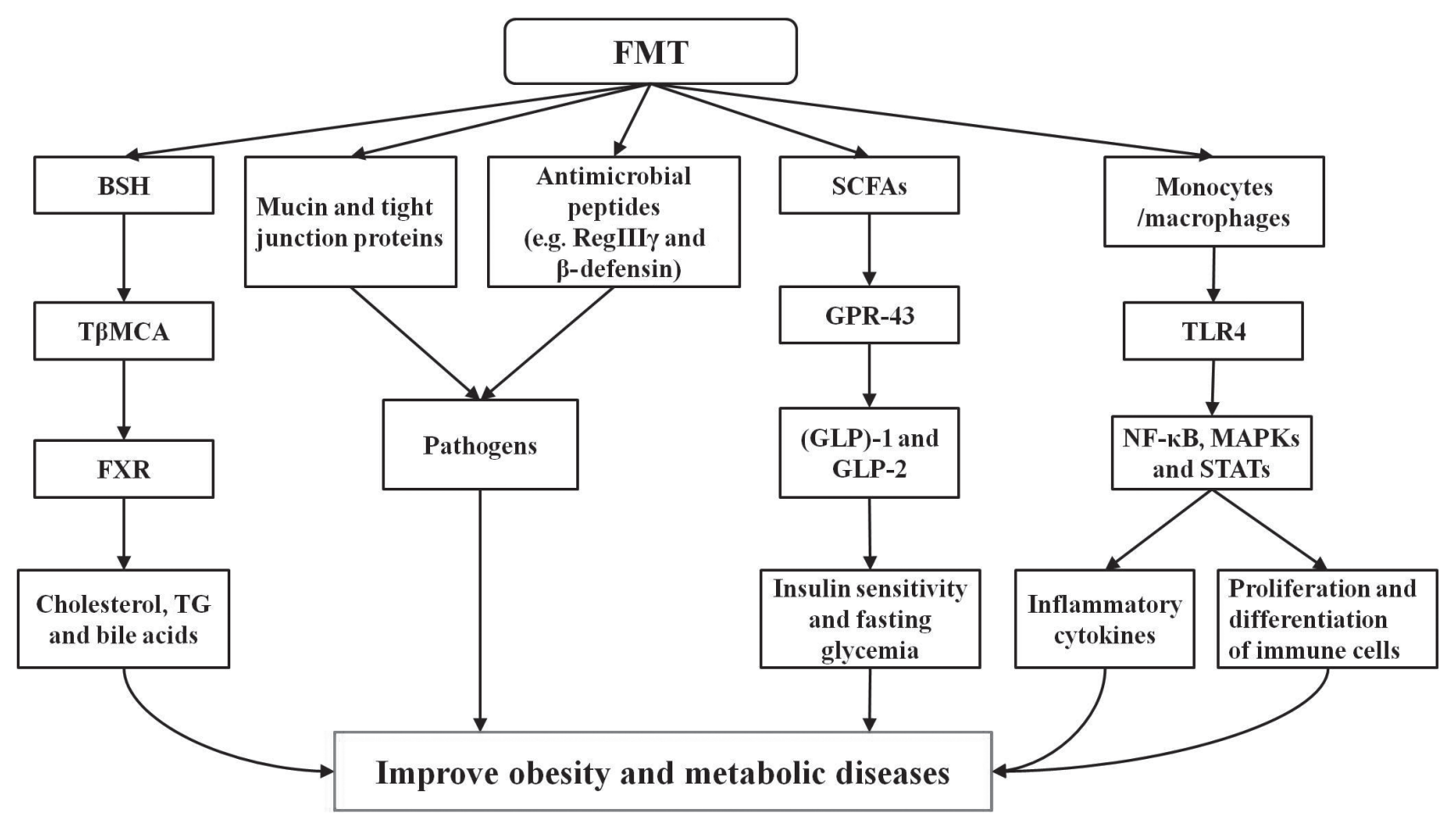

Figure 2. Possible mechanisms underlying the relationship between fecal microbiota transplantation (FMT) and obesity are as follows: FMT manipulates the composition of gut microbiota and thus bacterial bile salt hydrolase (BSH) enzymes in the gut influence host physiological processes by decreasing the abundance of T $\beta$ MCA (tauro- $\beta$-muricholic acid), a potent antagonist of the host bile receptor farnesoid X receptor (FXR), causing a decrease of body weight gain, plasma cholesterol levels, and liver triglycerides. Furthermore, FMT could inhibit growth of pathogens by stimulating secretion of antimicrobial peptides such as RegIIIg and $\beta$-defensin. FMT may be able to enhance the gut barrier by maintaining tight junctions and promoting mucin production. Bacterial derived short-chain fatty acids (SCFAs) activate G-protein coupled receptor (GPR)-43 and trigger secretion of glucagon-like peptide (GLP)-1 and GLP-2 that are involved in regulating fasting glycemia and affecting insulin sensitivity. FMT might also mediate immunomodulation via Toll-like receptor-4 (TLR4) on monocytes/macrophages, triggering the activation of nuclear factor-kappa B (NF- $\mathrm{B}$ ), mitogen activated protein kinase (MAPK), signal transducer and activator of transcription (STAT), which may also mediate inflammatory cytokine secretion and contribute to proliferation and differentiation of immune cells.

metabolic phenotypes than mice receiving their lean twin's gut commensals. Differences in body composition were correlated with differences in fermentation of short-chain fatty acids (increased in lean mice), metabolism of branched-chain amino acids (increased in obese mice), and microbial transformation of bile acid species (increased in lean mice and correlated with down-regulation of host farnesoid $\mathrm{X}$ receptor signaling). Because mice are coprophagic, the potential for transfer of intestinal bacteria through the fecal-oral route is high. Therefore, the authors used cohousing mice harboring an obese twin's microbiota $(\mathrm{Ob})$ with mice containing the lean co-twin's microbiota $(\mathrm{Ln})$ : they revealed that cohousing $\mathrm{Ln}$ and $\mathrm{Ob}$ mice prevented development of increased adiposity, body mass, and obesity-associated metabolic phenotypes in Ob cage mates and transformed their microbiota's metabolic profile to a leanlike state. Rescue correlated with invasion of specific members of Bacteroidetes from the Ln microbiota into $\mathrm{Ob}$ microbiota. These results reveal that transmissible and modifiable microbiota influences the host biology. Roux-en-Y gastric bypass (RYGB), which is a highly effective treatment for severe obesity and type 2 diabetes, is characterized by a marked and sustained loss of $\sim 65$ to $75 \%$ of excess body weight and fat mass. ${ }^{62}$ These effects are not only attributable to decreased caloric intake or absorption, but also to significant alterations of intestinal bacteria profiles. Numerous studies have shown that RYGB can change the composition of 
gut microbiota (Figure 3). ${ }^{46,63,64}$ Recently, Liou et a ${ }^{65}$ demonstrated that alterations of intestinal bacteria can contribute to reducing host weight and adiposity after RYGB surgery in mice. Compared with SHAM mice (diet-induced obese C57BL/6J mice are submitted to sham surgery), rapid loss of weight, reduction of adiposity, and improvement of glucose metabolism were observed in RYGB mice (diet-induced obese C57BL/6J mice are submitted to Roux-en-Y gastric bypass surgery). Furthermore, the levels of Verrucomicrobia (Akkermansia), Gammaproteobacteria (Escherichia), and Bacteroidales rapidly and sustainedly increased in RYGB mice. Transfer of gut microbiota from RYGB-treated mice to nonoperated, germ-free mice resulted in weight loss, decreased fat mass, and significantly reduced fasting triglyceride levels in the recipient animals (RYGB-R mice) relative to recipients of microbiota induced by sham surgery (SHAM-R mice). The relative abundance of Verrucomicrobiales remained markedly higher in RYGB-R mice than in SHAM-R mice. In addition, the enrichment of Akkermansia and Alistipes (Bacteroidetes phylum) in RYGB-R mice is similar to that of RYGB mice. In keeping with the latter observation, Zhang et al ${ }^{66}$ transplanted microbiotas from 16 obese juveniles and 16 controls separately into 64 germ-free male Swiss Webster mice caged in pairs and fed standard chow. They found that the average body weight gain of mice with microbiotas from obese donors was significantly higher than that of the control group. Meanwhile, total feeding efficiency was also higher in mice with "obese microbiota". Further research revealed that cecal concentrations of butyric acid and iso-butyric acid were significantly lower in the obese

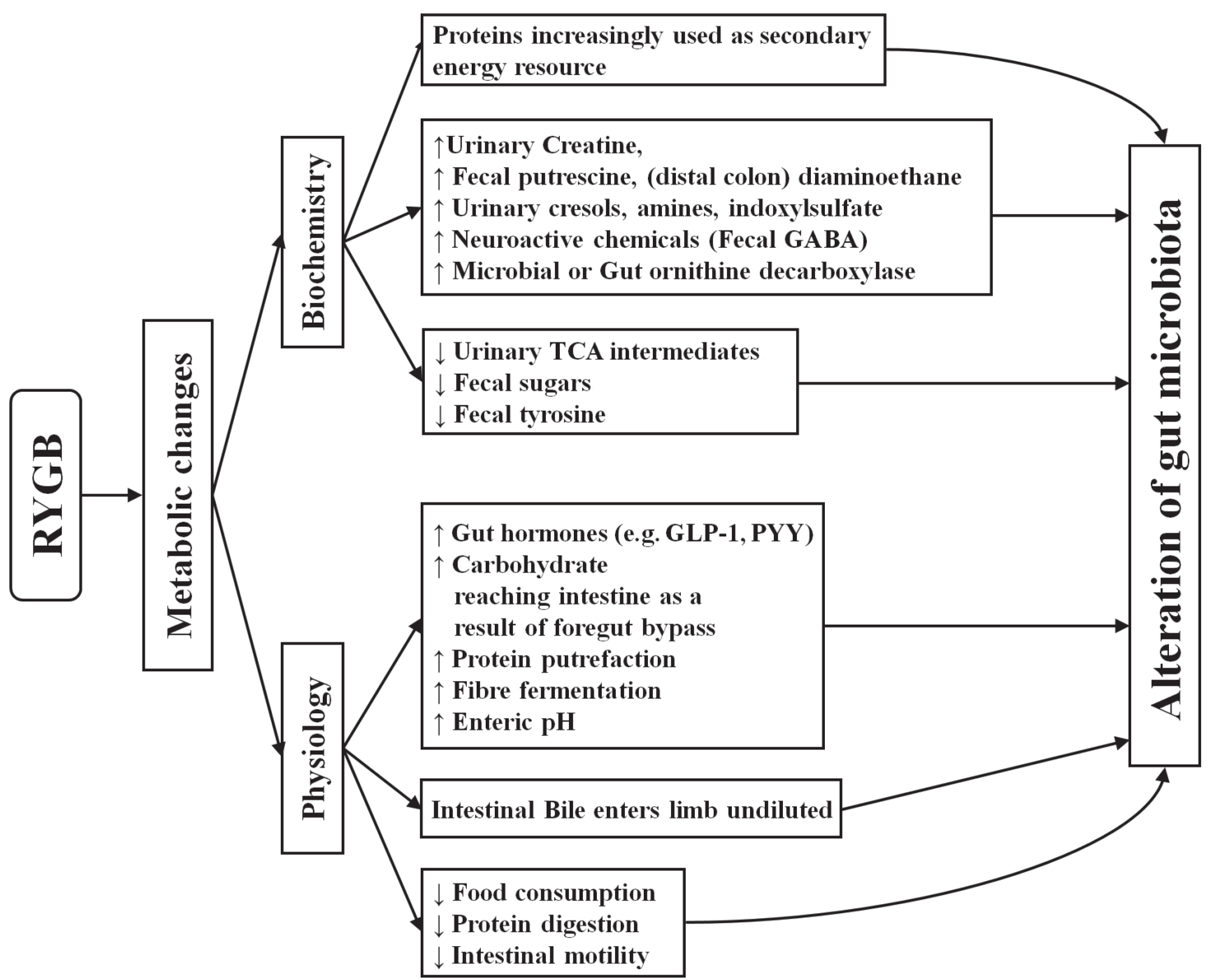

Figure 3. Metabolic Roux-en-Y gastric bypass surgery (RYGB) may impact upon the composition and regulatory activity of the microbiota by metabolic modulation. Abbreviations: GABA: g-aminobutyric acid; GLP-1: glucagonlike peptide-1; PYY: peptide YY; TCA: tricarboxylic acid. 
group, showing an altered microbial activity. These results establish gut microbiota as a causal factor for altered weight gain and changes in cecal levels of short-chain fatty acids. In conclusion, the above data clearly demonstrate the beneficial effects of FMT treatment in obesity and the metabolic syndrome.

\section{FMT SIDE EFFECTS}

Although the main advantage of FMT is that it has limited adverse effects, these are still an issue. At present, the most frequent are mild adverse effects related to abdominal discomfort (such as pain, bloating, nausea or even vomiting), but severe adverse effects have also been reported, including intestinal perforation, posttransplant sepsis or bacteremia. Despite these safety concerns related to transference of a complex and undefined living microbial community from one human to another, there is very little current information about the practice of FMT. Patients first need to be informed about the potential risks before consenting to the procedure. The optimal donor should be chosen based on his/her medical history and laboratory testing. A number of precautions and risks must be considered during donor selection. Potential donors should be screened for symptoms which may confer increased risk for transmitting infection. Furthermore, donors should be free of diseases or conditions which may, even theoretically, be transmissible by stool. Evaluating the potential risk of FMT with respect to possible future infections, autoimmune or metabolic diseases or even cancer are still required. Finally, in order to develop FMT therapy, a large number of human clinical trials need to be conducted.

\section{REGULATORY ISSUES}

There is an increasingly urgent need to regulate fecal microbiota transplantation owing to the remarkable interest that this therapy is generating. Regulation of FMT varies greatly between countries worldwide. In the United States, the US Food and Drug Administration (FDA) has determined that fecal microbiota administered via colonoscopy constitutes a biological product and a drug used to diagnose, cure, mitigate, treat or prevent disease or influence the structure or function of the body. ${ }^{67}$ The physician must obtain informed consent, make clear the risks and benefits of the procedure, and explain that it is an investigational therapy. In addition, the donor must be known to the patient or healthcare provider and the stool must be screened for pathogens. ${ }^{68}$ Similarly, Health Canada considers FMT and related synthetic stool therapies as a "New Biologic Drug" and requires that any clinical study go through the process of a clinical trial application to ensure that quality and safety standards are met. ${ }^{69}$ In the UK, where FMT has also been used to treat CDI, the treatment is considered safe and effective. ${ }^{70}$ However, in Europe the European Medicines Agency has not regulated FMT for CDI thus far, ${ }^{71}$ while in Australia, where FMT is not considered a drug or regulated for any indication, the Therapeutic Goods Administration has provided no communications regarding FMT. Though several FMT trials are ongoing in China, there has been no indication that the Ministry of Health intends to exercise authority over the procedure.

\section{CONCLUDING REMARKS}

Accumulating evidence has suggested that the maintenance of a healthy intestinal microbiota bacteria plays a pivotal role in preserving good health as well as avoiding the development of obesity and its associated metabolic disorders. ${ }^{72}$ However, although it is known that each specific species of gut microbiota can have either a pathogenic or protective role in the progression of obesity, ${ }^{73}$ a truly adequate longitudinal study of the exact relationship between intestinal commensal bacteria and the development of obesity has not as yet been carried out. It is thus obvious that in-depth studies on the mechanism(s) of interaction between gut microbiota and host need to be conducted in different populations, while in parallel, well-controlled clinical work with commensal bacteria is essential to guarantee a safe outcome in obese FMT patients. Over the past few decades, a large number of studies have provided a compelling rationale for exploring FMT as adjunctive therapy for obesity and the emergence of promising experimental studies has led to several clinical trials of FMT in human subjects. Meanwhile, increasing awareness and acceptance as regards the therapeutic use of FMT, partially due to its perception as a 'natural' treatment and its relatively inexpensive implementation, is also encouraging. ${ }^{74}$ Synergistic in vivo and in vitro studies must also be carried out 
to advance our knowledge in this field, translating the potentials for targeted therapy from preclinical models to humans. Future studies should focus on identification of optimal bacterial biomarkers for obesity diagnosis and therapeutic targets. Moreover, since this research has opened up an entirely new approach to the understanding and treatment of obesity, FMT may be considered a potentially useful therapy for additional conditions in the future.

\section{CONFLICT OF INTEREST}

The authors declare that they have no conflict of interest.

\section{ACKNOWLEDGEMENTS}

We thank Yue Cai for the critical review and important intellectual contributions to the final version of the manuscript.

\section{REFERENCES}

1. James WPT, 2008 The epidemiology of obesity: the size of the problem. J Intern Med 263: 336-352.

2. Shulman GI, 2014 Ectopic fat in insulin resistance, dyslipidemia, and cardiometabolic disease. NEJM 371 : 2236-2237.

3. Bouchard C, 2000 Inhibition of food intake by inhibitors of fatty acid synthase. NEJM 343: 1888-1889.

4. Kayser B, Verges S, 2013 Hypoxia, energy balance and obesity: from pathophysiological mechanisms to new treatment strategies. Obes Rev 14: 579-592.

5. Scarpellini E, Campanale M, Leone D, et al, 2012 Gut microbiota and obesity. Diges Dis 30: 196-200.

6. Savage DC, 2003 Microbial ecology of the gastrointestinal tract. Annu Rev Microbiol 31: 107-133.

7. Ursell LK, Haiser HJ, Treuren WV, et al, 2014 The Intestinal Metabolome: An Intersection Between Microbiota and Host. Gastroenterology 146: 1470-1476.

8. Sender R, Fuchs S, Milo R, 2016 Are We Really Vastly Outnumbered? Revisiting the Ratio of Bacterial to Host Cells in Humans. Cell 164: 337-340.

9. Turnbaugh PJ, Ley RE, Hamady M, et al, 2007 The human microbiome project: exploring the microbial part of ourselves in a changing world. Nature 449: 804-810.

10. Zaneveld J, Turnbaugh PJ, Lozupone C, et al, 2008 Host-bacterial coevolution and the search for new drug targets. Curr Opin Chem Biol 12: 109-114.

11. Mackie RI, Sghir A, Gaskins HR, 1999 Developmental microbial ecology of the neonatal gastrointestinal tract.
Am J Clin Nutr 69: 1035s-1045s.

12. Claesson MJ, Cusack S, O'Sullivan O, et al, 2011 Composition, variability, and temporal stability of the intestinal microbiota of the elderly. Proc Natl Acad Sci USA 108(Suppl 1): 4586-4591.

13. Zoetendal EG, Akkermans AD, De Vos WM, 1998 Temperature gradient gel electrophoresis analysis of 16S rRNA from human fecal samples reveals stable and host-specific communities of active bacteria. Appl Environ Microbiol 64: 3854-3859.

14. Koren O, Spor A, Felin J, et al, 2011 Human oral, gut, and plaque microbiota in patients with atherosclerosis. Proc Natl Acad Sci USA 108(Suppl 1): 4592-4598.

15. Koren O, Goodrich JK, Cullender TC, et al, 2012 Host remodeling of the gut microbiome and metabolic changes during pregnancy. Cell 150: 470-480.

16. Abt MC, Artis D, 2009 The intestinal microbiota in health and disease: the influence of microbial products on immune cell homeostasis. Curr Opin Gastroen 25: 496-502.

17. Tremaroli V, Bäckhed F, 2012 Functional interactions between the gut microbiota and host metabolism. Nature 489: 242-249.

18. Vinolo MAR, Rodrigues HG, Nachbar RT, et al, 2011 Regulation of Inflammation by Short Chain Fatty Acids. Nutrients 3: 858-876.

19. Zaibi MS, Stocker CJ, O'Dowd J, et al, 2010 Roles of GPR41 and GPR43 in leptin secretory responses of murine adipocytes to short chain fatty acids. FEBS Lett 584: 2381-2386.

20. Cox MA, Jackson J, Stanton M, et al, 2009 Shortchain fatty acids act as antiinflammatory mediators by regulating prostaglandin $\mathrm{E}$ and cytokines. World $\mathrm{J}$ Gastroentero 15: 5549-5557.

21. Luster AD, Alon R, von Andrian UH, 2005 Immune cell migration in inflammation: present and future therapeutic targets. Nat Immunol 6: 1182-1190.

22. Aoyama M, Kotani J, Usami M, 2010 Butyrate and propionate induced activated or non-activated neutrophil apoptosis via HDAC inhibitor activity but without activating GPR-41/GPR-43 pathways. Nutrition 26: 653-661.

23. Ramos MG, Rabelo FL, Duarte T, et al, 2002 Butyrate induces apoptosis in murine macrophages via caspase-3, but independent of autocrine synthesis of tumor necrosis factor and nitric oxide. Braz J Med Biol Res 35: 161173.

24. Kuritaochiai T, Ochiai K, Fukushima K, 2001 Butyric Acid-Induced T-Cell Apoptosis Is Mediated by Caspase-8 and -9 Activation in a Fas-Independent Manner. Clin Diagn Lab Immunol 8: 325-332.

25. Bailón E, Cueto-Sola M, Utrilla P, et al, 2010 Butyrate in vitro immune-modulatory effects might be mediated through a proliferation-related induction of apoptosis. Immunobiology 215: 863-873.

26. Chassaing B, Kumar M, Baker MT, et al, 2014 Mam- 
malian gut immunity. Biomed J 37: 246-258.

27. Salminen S, Bouley C, Boutronruault MC, et al, 1998 Functional food science and gastrointestinal physiology and function. Brit J Nutr 80(Suppl 1): S147-S171.

28. Tsai F, Coyle WJ, 2009 The microbiome and obesity: is obesity linked to our gut flora? Curr Gastroen Rep 11: 307-313.

29. Bäckhed F, Ding H, Wang T, et al, 2004 The gut microbiota as an environmental factor that regulates fat storage. Proc Natl Acad Sci USA 101: 15718-15723.

30. Bäckhed F, Manchester JK, Semenkovich CF, et al, 2007 Mechanisms underlying the resistance to dietinduced obesity in germ-free mice. Proc Natl Acad Sci USA 104: 979-984.

31. Turnbaugh PJ, Ley RE, Mahowald MA, et al, 2006 An obesity-associated gut microbiome with increased capacity for energy harvest. Nature 444: 1027-1031.

32. Ley RE, Backhed F, Turnbaugh P, et al, 2005 Obesity alters gut microbial ecology. Proc Natl Acad Sci USA 102: 11070-11075.

33. Wellen KE, Hotamisligil GS, 2005 Inflammation, stress, and diabetes. J Clin Invest 115: 1111-1119.

34. Dibaise JK, Zhang H, Crowell MD, et al, 2008 Gut Microbiota and Its Possible Relationship With Obesity. Mayo Clin Proc 83: 460-469.

35. Cani PD, Amar J, Iglesias MA, et al, 2007 Metabolic endotoxemia initiates obesity and insulin resistance. Diabetes 56: 1761-1772.

36. Pappo I, Becovier H, Berry EM, et al, 1991 Polymyxin $B$ reduces cecal flora, TNF production and hepatic steatosis during total parenteral nutrition in the rat. $\mathrm{J}$ Surg Res 51: 106-112.

37. Ley RE, Turnbaugh PJ, Klein S, et al, 2007 Microbial ecology: Human gut microbes associated with obesity. Nature 444: 1022-1023.

38. Turnbaugh PJ, Hamady M, Yatsunenko T, et al, 2009 A core gut microbiome in obese and lean twins. Nature 457: 480-484.

39. Walters WA, Xu Z, Knight R, 2014 Meta-analyses of human gut microbes associated with obesity and IBD. Febs Lett 588: 4223-4233.

40. Kocełak P, Zakgołąb A, Zahorskamarkiewicz B, et al, 2013 Resting energy expenditure and gut microbiota in obese and normal weight subjects. Eur Rev Med Pharmacol Sci 17: 2816-2821.

41. Faith JJ, Guruge JL, Charbonneau M, et al, 2013 The Long-Term Stability of the Human Gut Microbiota. Science 341: 1237439.

42. Jumpertz R, Le DS, Turnbaugh PJ, et al, 2011 Energybalance studies reveal associations between gut microbes, caloric load, and nutrient absorption in humans. Am J Clin Nutr 94: 58-65.

43. Cotillard A, Kennedy SP, Kong LC, et al, 2013 Dietary intervention impact on gut microbial gene richness. Nature 500: 585-588.

44. Le CE, Nielsen T, Qin J, et al, 2013 Richness of human gut microbiome correlates with metabolic markers. Nature 500: 541-546.

45. Everard A, Belzer C, Geurts L, et al, 2013 Cross-talk between Akkermansia muciniphila and intestinal epithelium controls diet-induced obesity. Proc Natl Acad Sci USA 110: 9066-9071.

46. Zhang H, Dibaise JK, Zuccolo A, et al, 2009 Human gut microbiota in obesity and after gastric bypass. Proc Natl Acad Sci USA 106: 2365-2370.

47. Kang Y, Zhang X, Cai Y, et al, 2016 Gut microbiota and metabolic disease: from pathogenesis to new therapeutic strategies. Rev Med Microbiol 27: 141-152.

48. Fei N, Zhao L, 2013 An opportunistic pathogen isolated from the gut of an obese human causes obesity in germfree mice. Isme J 7: 880-884.

49. Cani PD, Amar J, Iglesias MA, et al, 2007 Metabolic endotoxemia initiates obesity and insulin resistance. Diabetes 56: 1761-1772.

50. Sanders WE, Jr., Sanders CC, 1997 Enterobacter spp.: pathogens poised to flourish at the turn of the century. Clin Microbiol Rev 10: 220-241.

51. Bakken JS, Borody T, Brandt LJ, et al, 2011 Treating Clostridium difficile Infection With Fecal Microbiota Transplantation. Clin Gastroenterol H 9: 1044-1049.

52. Smits LP, Bouter KEC, Vos WM, et al, 2013 Therapeutic potential of fecal microbiota transplantation. Gastroenterology 145: 946-953.

53. Gupta S, Allen-Vercoe E, Petrof EO, 2016 Fecal microbiota transplantation: in perspective. Therap Adv Gastroenterol 9: 229-239.

54. Borody TJ, Khoruts A, 2011 Fecal microbiota transplantation and emerging applications. Nat Rev Gastro Hepat 9: 88-96.

55. Kang Y, Cai Y, 2017 Gut microbiota and hepatitis-Bvirus-induced chronic liver disease: implications for faecal microbiota transplantation therapy. J Hosp Infect 96: 342-348.

56. Vrieze A, Nood EV, Holleman F, et al, 2012 Transfer of Intestinal Microbiota From Lean Donors Increases Insulin Sensitivity in Individuals With Metabolic Syndrome. Gastroenterology 143: 913-916 (e917).

57. Duncan S, Belenguer A, Holtrop G, et al, 2007 Reduced dietary intake of carbohydrates by obese subjects results in decreased concentrations of butyrate and butyrateproducing bacteria in feces. Appl Environ Microb 73: 1073-1078.

58. Hamer HM, Jonkers D, Venema K, et al, 2008 Review article: the role of butyrate on colonic function. Aliment Pharm Therap 27: 104-119.

59. Louis P, Flint HJ, 2009 Diversity, metabolism and microbial ecology of butyrate-producing bacteria from the human large intestine. Fems Microbiol Lett 294: $1-8$.

60. Vanhoutvin SALW, Troost FJ, Kilkens TOC, et al, 2009 The effects of butyrate enemas on visceral perception in healthy volunteers. Neurogastroenterol Motil 21: 
952-e976.

61. Ridaura VK, Faith JJ, Rey FE, et al, 2013 Gut microbiota from twins discordant for obesity modulate metabolism in mice. Science 341: 1241214.

62. Hatoum IJ, Greenawalt DM, Cotsapas C, et al, 2011 Heritability of the weight loss response to gastric bypass surgery. J Clin Endocr Metab 96: 1630-1633.

63. Jia VL, 2011 Metabolic surgery profoundly influences gut microbial-host metabolic cross-talk. Gut 60: 12141223.

64. Furet JP, Kong LC, Tap J, et al, 2010 Differential Adaptation of Human Gut Microbiota to Bariatric Surgery-Induced Weight Loss: Links With Metabolic and Low-Grade Inflammation Markers. Diabetes 59: 3049-3057.

65. Liou AP, Paziuk M, Jr., LJ, et al, 2013 Conserved shifts in the gut microbiota due to gastric bypass reduce host weight and adiposity. Sci Transl Med 5: 178ra141.

66. Zhang L, Bahl MI, Hellgren L, et al, 2015 Obesityassociated fecal microbiota from human modulates body mass and metabolites in mice. EMBL Conference 2015, Heidelberg, Germany.

67. U.S. Food and Drug Administration, FDA Drug Definition. FDA Glossary of Terms. Accessed December 14 2016; http://www.fda.gov/drugs/informationon drugs/
ucm079436.htm\#D.

68. FDA, 2014 Draft Guidance for Industry: Enforcement Policy Regarding Investigational New Drug Requirements for Use of Fecal Microbiota for Transplantation to Standard Therapies. Silver Sping, MD: US Food and Drug Administration.

69. Allen-Vercoe E, Reid G, Viner N, et al, 2012 A Canadian Working Group report on fecal microbial therapy: microbial ecosystems therapeutics. Can J Gastroenterol 26: 457-462.

70. Smith, Mark B, Kelly, et al, 2014 Policy: How to regulate faecal transplants. Nature 506: 290-291.

71. Van NE, Speelman P, Nieuwdorp M, et al, 2014 Fecal microbiota transplantation: facts and controversies. Curr Opin Gastroen 30: 34-39.

72. Kang Y, Cai Y, Zhang X, et al, 2016 Altered gut microbiota in RA: implications for treatment. Z Rheumatol 76: 451-457.

73. Kang YB, Cai Y, Zhang H, 2016 Gut microbiota and allergy/asthma: From pathogenesis to new therapeutic strategies. Allergol Immunopathol 45: 305-309.

74. Kelly CR, Kahn S, Kashyap P, et al, 2015 Update on Fecal Microbiota Transplantation 2015: Indications, Methodologies, Mechanisms, and Outlook. Gastroenterology 149: 223-237. 